\title{
ON THE GEOMETRY OF EXTENSIONS OF IRREDUCIBLE MODULES FOR SIMPLE ALGEBRAIC GROUPS
}

\author{
STEPHen R. Doty and John B. Sullivan
}

\begin{abstract}
Let $G$ be a simple, simply connected affine algebraic group over an algebraically closed field $k$ of non-zero characteristic $p$. We consider the problem of determining the extensions of irreducible modules by irreducible modules. The extensions may be realized as submodules of modules induced from characters on a Borel subgroup of $G$. The geometry of the distribution of composition factors of those induced modules is determined by an operation (namely, alcove transition) of the Weyl group on the space of weights. Generically in the lowest $p^{2}$-alcove, that operation stabilizes a canonical subset of the set of highest weights of those irreducible modules which extend the irreducible module of some fixed highest weight. The stability leads to an upper bound on that subset, which can be refined using the translation principle. We give a conjecture for the generic distribution of extensions of irreducible modules by a fixed irreducible module.
\end{abstract}

Introduction. Let $G$ be a simple, simply connected affine algebraic group over an algebraically closed field $k$ of non-zero characteristic $p$. $(B, T)$ is a fixed Borel subgroup and maximal torus pair, $X(B)$ is the character group of $B$, and $B^{\text {opp }}$ is the opposite Borel subgroup. Take the positive roots of $(G, T)$ to be the roots of $\left(B^{\text {opp }}, T\right)$. Let $G_{1}$ be the kernel of the Frobenius morphism of $G$. Let $\left\{H^{i}(\chi)\right\}_{i=0}^{\operatorname{dim} G / B}$ $=\left\{H^{i}(G / B, L(\chi))\right\}_{i=0}^{\operatorname{dim} G / B}$ be the sheaf cohomology modules of the homogeneous space $G / B$ at the line bundle $L(\chi)$ induced from a character $\chi$ on $B$.

For each dominant character $\lambda, H^{0}(\lambda)$ has as its socle the irreducible module $M_{\lambda}$ of highest weight $\lambda$. The formal character of $M_{\lambda}$ can be computed in terms of the formal characters of the modules $\left\{M_{\mu} \mid \mu \neq \lambda, \mu\right.$ strongly linked to $\lambda\}$, once the multiplicities $\left[H^{0}(\lambda): M_{\mu}\right]$ of the $M_{\mu}$ as composition factors of $H^{0}(\lambda)$ are known. Let $X\left[H^{0}(\lambda)\right]=\{(\mu, n) \in$ $\left.X(B) \times \mathbf{Z}_{\geq 0} \mid\left[H^{0}(\lambda): M_{\mu}\right]=n\right\}$, let $X_{\lambda}=\left\{\mu \in X(B) \mid\left[H^{0}(\lambda): M_{\mu}\right] \neq\right.$ $0\}$, and let $X^{\lambda}=\left\{\mu \in X(B) \mid\left[H^{0}(\mu): M_{\lambda}\right] \neq 0\right\}$. In [7], the authors defined the $W$-linkage class $W L \cdot \lambda$ of a character $\lambda$. They showed that $W L \cdot \lambda$ is an upper bound for $X_{\lambda}$, when $\lambda$ is a weight generic in the 
lowest $p^{2}$-alcove, and conjectured that $W L \cdot \lambda=X_{\lambda}$. In 1.2, we prove that conjecture, using a Theorem of J.-c. Ye [9]. In this way, we can tell when $\left[H^{0}(\lambda): M_{\mu}\right]$ is non-zero, although we are not in a position to give the value of the multiplicity $\left[H^{0}(\lambda): M_{\mu}\right]$.

Let $v$ be a special vertex $(\S 0)$. Let $W_{v}$ be the subgroup of the affine Weyl group that fixes $v . W_{v}$ is canonically isomorphic to the Weyl group $W$; let $y$ be the element of $W_{v}$ corresponding to an element $w$ of $W$. Let Soc $^{j} M$ be the $j$ th socle term for a module $M$. Let $\lambda$ be a weight, generic in the lowest $p^{2}$-alcove. The $W$-linkage class of $\lambda$, as a bound on $X_{\lambda}$, arose in [7] in connection with the bearing that the group of alcove transition operators $\left\{I_{y}\right\}_{y \in W_{v}}$ has on the supposed structural similarity between $H^{l(w)}(w \cdot \lambda)$ and $H^{0}(y \cdot \lambda)$. In fact, the operator $I_{y}$ transforms $X\left[H^{l(w)}(w \cdot \lambda)\right]$ into $X\left[H^{0}(y \cdot \lambda)\right]$, and $X\left[\operatorname{Soc}^{1}\left(H^{l(w)}(w \cdot \lambda)\right)\right]$ into $X\left[\operatorname{Soc}^{1}\left(H^{0}(y \cdot \lambda)\right)\right]$. Pursuing the theme of structural similarity further, we show in 1.3 that $I_{y}$ transforms $X\left[\operatorname{Soc}^{2}\left(H^{l(w)}(w \cdot \lambda)\right)\right]$ into $X\left[\operatorname{Soc}^{2}\left(H^{0}(y \cdot \lambda)\right)\right]$. As a corollary, we show that

$$
H^{l(w)}\left(G / B G_{1}, L\left(\operatorname{Soc}^{2}\left(Z_{1}(w \cdot \lambda)\right)\right)\right)=\operatorname{Soc}^{2}\left(H^{l(w)}(w \cdot \lambda)\right),
$$

where $Z_{1}(w \cdot \lambda)$ is the $T G_{1}$-module induced from the $T B_{1}$-module $k w \cdot \lambda$. In Andersen's work [3], the question arises of whether the socle series of $Z_{1}(w \cdot \lambda)$ can be lifted to the socle series for $H^{l(w)}(w \cdot \lambda)$. That Corollary gives an affirmative answer for $j=2$.

Let $\lambda$ be a weight in the box $V^{v}$ (see $\S 0$ ). Each weight $\mu$ has a unique expression $\mu=\mu^{0}+p \mu^{1}$, where $\mu^{0} \in V^{(p-1) \rho}$ and $\mu^{1} \in X(T)$. In this paper, we handle extensions only between irreducible modules $M_{\mu}$ and $M_{\lambda}$ where $\mu^{0} \neq \lambda^{0}$. (The case $\mu^{0}=\lambda^{0}$ can be studied separately using the fact that $\operatorname{Ext}_{G}^{1}\left(M_{\lambda^{0}+p \mu^{1}}, M_{\lambda^{0}+p \lambda^{1}}\right) \cong \operatorname{Ext}_{G}^{1}\left(\mu^{1}, \lambda^{1}\right)$, for $\lambda$ not on a wall of the dominant chamber.) In [2], Andersen showed that $I_{W_{v}}$ stabilizes the set $m \operatorname{Ext}(\lambda)^{0}=\left\{(\mu, n) \in X(B) \times \mathbf{Z}_{\geq 0} \mid \mu^{0} \neq \lambda^{0}\right.$ and $n=$ $\left.\operatorname{dim}_{k} \operatorname{Ext}_{G}^{1}\left(M_{\mu}, M_{\lambda}\right)\right\}$, for $\lambda$ generic in the lowest $p^{2}$-alcove. In $\S 2$, we study the geometry of the operation of $I_{W_{v}}$ on the $\operatorname{set} \operatorname{Ext}(\lambda)^{0}=\{\mu \in$ $X(T) \mid \mu^{0} \neq \lambda^{0}$ and $\left.\operatorname{Ext}_{G}^{1}\left(M_{\mu}, M_{\lambda}\right) \neq 0\right\} . \operatorname{Ext}(\lambda)^{0}$ is contained in the union of $X_{\lambda}$ and $X^{\lambda}$. The $I_{W_{v}}$-orbits in $\operatorname{Ext}(\lambda)^{0}$ fall into three classes: (i) the orbits that lie in $X_{\lambda}$, (ii) the orbits that lie in $X^{\lambda}$, (iii) the orbits that meet both $X_{\lambda}$ and $X^{\lambda}$. From each orbit, we pick out an element which is specially situated relative to $v$, depending on the class to which the orbit belongs. From an orbit in the third class, the element is $s_{\beta *} \cdot \lambda$, where $\beta$ is a simple root and $s_{\beta^{*}} \cdot \lambda$ is the reflection of $\lambda$ in the $\beta$ affine hyperplane which is below $\lambda$ and nearest to $\lambda$. These considerations lead to the Theorem in 2.2, which gives an upper bound on $\operatorname{Ext}(\lambda)^{0}$ as a union of certain $I_{w_{v}}$-orbits in $X(T)$. 
In $\S \S 2.3$ and 2.4 , we consider the question of whether $s_{\beta *} \cdot \lambda, \beta$ simple, lies in $\operatorname{Ext}(\lambda)$. In particular, we show that when $\lambda$ lies in a translate of the fundamental alcove, $\operatorname{Soc}^{2}\left(H^{0}(\lambda)\right) / \operatorname{Soc}^{1}\left(H^{0}(\lambda)\right) \cong$ $\oplus_{\beta \text { simple }} M_{s_{\beta *}} \cdot \lambda$.

In $\S 3$, we show that the alcoves of characters $\chi$ and $\lambda$ must be compatibly oriented in order for $\chi$ to be an element of $\operatorname{Ext}(\lambda)$. This gives a tightening of the upper bound on the set $\operatorname{Ext}(\lambda)^{0}$, given in 2.2. Finally, in $\S 4$, we give a conjectural formula for $m \operatorname{Ext}(\lambda)^{0}$ in terms of the second and $[\operatorname{dim} G / B-1]$ st-socle layers of $H^{0}(\lambda)$.

We thank H. H. Andersen for discussing with us his work [3] during his visit to the University of Washington in the Summer, 1985.

0. Let $G$ be a simple, simply connected algebraic group over an algebraically closed field of characteristic $p$. Let $B$ be a Borel subgroup of $G$, with maximal torus $T$ and character group $X(B)=X(T)$. Let $B^{\text {opp }}$ be the Borel subgroup opposite to $B, R$ the set of roots for $(G, T), R^{+}$the set of roots for ( $\left.B^{\text {opp }}, T\right), S$ the set of simple roots in $R^{+}$, and $\rho$ the half sum of the positive roots. Let $R(T)$ be the root lattice in $X(T)$, and let $X(T)^{+}$be the set of dominant weights relative to $R^{+} . W$ is the Weyl group of $(G, T), s_{\gamma}$ is the reflection associated to a root $\gamma, l()$ the length function on $W$, and $w_{0}$ the longest word in $W .($, ) is a $W$-invariant inner product on $X(R) \otimes_{\mathrm{Z}} \mathbf{R}$, and $\alpha^{\nu}=2 \alpha /(\alpha, \alpha)$ is the coroot of $\alpha \in R$. Let $N=\operatorname{dim} G / B=l\left(w_{0}\right) . G_{1}$ is the kernel of the Frobenius map on $G$.

Notation. Much of this notation appears already in [7].

Let $T_{\chi}$ be the operator on $X(T)$ which translates each weight by a fixed weight $\chi$.

$$
\begin{aligned}
& K(p R(T))=\left\{T_{p^{\lambda}} \mid \lambda \in R(T)\right\} \text { is the root translation group. } \\
& K(p X(T))=\left\{T_{p \lambda} \mid \lambda \in X(T)\right\} \text { is the weight translation group. }
\end{aligned}
$$

$W_{p}=W \cdot K(p R(T))$ is the affine Weyl group. $W \cdot K(p X(T))$ augments $W_{p}$ by the fundamental group of $G . w(\chi)$ is the ordinary action of $w \in W_{p}$ on $\chi \in X(T)$, and $w \cdot \chi$ is the action $w \cdot \chi=w(\chi+\rho)-\rho$. $w_{1} w_{2} \cdot \chi$ is understood to mean $\left(w_{1} w_{2}\right) \cdot \chi$, for $w_{1}, w_{2} \in W_{p}$.

Let $\alpha \in R^{+}$and $n \in \mathbf{Z} . H_{\alpha, n}$ is the affine hyperplane $\{\chi \in X(T)$ $\left.\otimes_{\mathbf{Z}} \mathbf{R} \mid\left(\alpha^{\nu}, \chi+\rho\right)=n p\right\} . s_{\alpha, n p}$, the reflection operator in $H_{\alpha, n p}$, is an element of $W_{p}$.

A special vertex $v$ is an element of $X(T)$ of the form $\bigcap_{\beta \in S ; n_{\beta} \in \mathbf{Z}} H_{\beta, n_{\beta} p}$. $v$ is a common point for $N$ hyperplanes, one for each positive root. 
$W_{v}=\left\{\sigma \in W_{p} \mid \sigma \cdot v=v\right\}$ is the Weyl group at $v . W$ itself is the Weyl group at $-\rho$, and $W$ and $W_{v}$ are isomorphic via conjugation by $T_{v+\rho} . w$ and $y$ denote elements of $W$ and $W_{v}$ which correspond under the isomorphism. In particular, $y_{0} \in W_{v}$ corresponds to $w_{0}$, and $y_{\gamma} \in W_{v}$ corresponds to $s_{\gamma}$.

$$
F_{v}=\left\{\chi \in X(T) \mid n_{\beta} p \leq\left(\beta^{\nu}, \chi+\rho\right), \beta \in S\right\}
$$

is the dominant chamber at $v$.

$$
F^{v}=y_{0} \cdot F_{v}=\left\{\chi \in X(T) \mid n_{\beta} p \geq\left(\beta^{\nu}, \chi+\rho\right), \beta \in S\right\}
$$

is the negative dominant chamber at $v$.

$$
V_{v}=\left\{\chi \in X(T) \mid n_{\beta} p \leq\left(\beta^{\nu}, \chi+\rho\right)<\left(n_{\beta}+1\right) p, \beta \in S\right\}
$$

is the upper box at $v$.

$$
V^{v}=y_{0} \cdot V_{v}=\left\{\chi \in X(T) \mid\left(n_{\beta}-1\right) p<\left(\beta^{\nu}, \chi+\rho\right) \leq n_{\beta} p, \beta \in S\right\}
$$

is the lower box at $v$.

$V^{v}$ and $V_{v}$ are two fundamental domains for the operation of $K(p X(T))$ on $X(T)$. In particular, we may write each weight $\lambda$ as $\lambda=\lambda^{0}+p \lambda^{1}$, where $\lambda^{0} \in V^{(p-1) \rho}$ and $\lambda^{1} \in X(T)$.

0.1 . The extended affine Weyl group $W \cdot K(p X(T))$ operates on the set of hyperplanes $\left\{H_{\gamma, n p} \mid \gamma \in R^{+}, n \in \mathbf{Z}\right\}$ and on the set of special vertices.

Let $\gamma \in R$. The nearest lower $\gamma$-hyperplane reflection $s_{\gamma *}$ on $X(T)$ is defined by $s_{\gamma *} \cdot \lambda=s_{\gamma, n p} \cdot \lambda$, where $n p \leq\left(\gamma^{\nu}, \lambda+\rho\right)<(n+1) p . s_{\gamma *}$ operates trivially on the set of special vertices.

Let SL be the monoid of operators on $X(T)$ generated by the set $\left\{s_{\gamma *} \mid \gamma \in R^{+}\right\}$, including the identity. For each $p$-regular weight $\lambda$, let SL $\cdot \lambda$ be the set of weights into which $\lambda$ can be moved by the elements of SL. Write $\chi \uparrow \lambda$ ( $\chi$ is strongly linked to $\lambda$ ) if $\chi \in \mathrm{SL} \cdot \lambda$.

The nearest upper $\gamma$-hyperplane reflection $s_{\gamma}^{*}$ on $X(T)$ is defined by $s_{\gamma}^{*} \cdot \lambda=s_{\gamma, n p} \cdot \lambda$, where $(n-1) p<\left(\gamma^{\nu}, \lambda+\rho\right) \leq n p$. Let $\mathrm{SL}^{-1}$ be the monoid of operators on $X(T)$ generated by the set $\left\{s_{\gamma}^{*} \mid \gamma \in R^{+}\right\}$, including the identity $\chi \in \mathrm{SL} \cdot \lambda$ and $\lambda \in \mathrm{SL}^{-1} \cdot \chi$ are equivalent.

0.2. Extensions. Let $M_{\lambda}$ be the irreducible $G$-module of highest (dominant) weight $\lambda$. Let $\left[M: M_{\lambda}\right]$ be the multiplicity of $M_{\lambda}$ as a composition factor of a module $M$. Let $H^{\prime}(\chi)$ be the $i$ th-cohomology 
module $H^{\prime}(G / B, L(\chi))$ for the line bundle $L(\chi)$ induced from a character $\chi$ on $B$. For a $B$-module $E$, we employ also the notation $H^{i}(G / B, E)$ for the cohomology module $H^{\prime}(G / B, L(E))$.

Definitions. Let $\lambda$ be a dominant character.

$$
\begin{aligned}
& X_{\lambda}=\left\{\chi \in X(T)^{+} \mid\left[H^{0}(\lambda): M_{\chi}\right] \neq(0)\right\}, \\
& X^{\lambda}=\left\{\chi \in X(T)^{+} \mid\left[H^{0}(\chi): M_{\lambda}\right] \neq(0)\right\} .
\end{aligned}
$$

$\chi \in X_{\lambda}$ and $\lambda \in X^{\chi}$ are equivalent.

Let $\operatorname{Ext}_{G}^{1}\left(M_{\chi}, M_{\lambda}\right)$ be the group of extension classes of $M_{\chi}$ by $M_{\lambda}$.

Definition. $\operatorname{Ext}(\lambda)=\left\{\chi \in X(T)^{+} \mid \operatorname{Ext}_{G}^{1}\left(M_{\chi}, M_{\lambda}\right) \neq(0)\right\}$.

$\chi$ and $\lambda$ are symmetrically related by $\operatorname{Ext}: \chi \in \operatorname{Ext}(\lambda)$ and $\lambda \in \operatorname{Ext}(\chi)$ are equivalent. See [6], for instance.

Definition. $\operatorname{Ext}(\lambda)^{0}=\left\{\chi \in \operatorname{Ext}(\lambda) \mid \chi^{0} \neq \lambda^{0}\right\}$.

Let $\mathbf{N}$ be the set of non-negative integers. The following definitions bring in multiplicities.

DEFINITIONS

$$
\begin{aligned}
m X_{\lambda} & =\left\{(\chi, n) \in X(T)^{+} \times \mathbf{N} \mid n=\left[H^{0}(\lambda): M_{\chi}\right]\right\}, \\
m X^{\lambda} & =\left\{(\chi, n) \in X(T)^{+} \times \mathbf{N} \mid n=\left[H^{0}(\chi): M_{\lambda}\right]\right\}, \\
m \operatorname{Ext}(\lambda) & =\left\{(\chi, n) \in X(T)^{+} \times \mathbf{N} \mid n=\operatorname{dim}_{k} \operatorname{Ext}_{G}^{1}\left(M_{\chi}, M_{\lambda}\right)\right\} .
\end{aligned}
$$

$(\chi, n) \in m X^{\lambda}$ and $(\lambda, n) \in m X_{\chi}$ are equivalent, as are $(\chi, n) \in m \operatorname{Ext}(\lambda)$ and $(\lambda, n) \in m \operatorname{Ext}(\chi)$.

Definition. $m \operatorname{Ext}(\lambda)^{0}=\left\{(\chi, n) \in m \operatorname{Ext}(\lambda) \mid \chi^{0} \neq \lambda^{0}\right\}$.

0.3. For each $y \in W_{p}$, define the alcove transition operator $I_{y}$ on $X(T)$ by letting $I_{y}=T_{y \cdot v-v}$ on each box $V^{v}$. I: $W_{p} \rightarrow \operatorname{Perm}(X(T))$, $y \rightarrow I_{y}$, is a homomorphism of groups, since $I_{y} \cdot V^{v}=V^{y \cdot v}$. The box $V^{v}$ is fixed pointwise by the subgroup of operators $I_{W_{v}}=\left\{I_{y} \mid y \in W_{v}\right\}$. That definition agrees with the definition of the alcove transition operators given in [7]. 
DEFINITIONS

$W L \cdot \lambda=\bigcap_{y \in W_{p}} I_{y^{-1}} \cdot \mathrm{SL} \cdot(y \cdot \lambda)$ is the $W$-linkage class of $\lambda$. $W L^{-1} \cdot \lambda=\bigcap_{y \in W_{p}} y^{-1} \cdot \mathrm{SL}^{-1} \cdot\left(I_{y} \cdot \lambda\right)$ is the inverse $W$-linkage class of $\lambda$. $\chi \in W L \cdot \lambda$ and $\lambda \in W L^{-1} \cdot \chi$ are equivalent.

By [7], for each special vertex $v, W L \cdot \lambda=\bigcap_{y \in W_{v}} I_{y^{-1}} \cdot \operatorname{SL} \cdot(y \cdot \lambda)$ and $W L^{-1} \cdot \lambda=\bigcap_{y \in W_{v}} y^{-1} \cdot \mathrm{SL}^{-1} \cdot\left(I_{y} \cdot \lambda\right)$. In particular, when $\lambda \in V^{v}$, we have $W L^{-1} \cdot \dot{\lambda}=\bigcap_{y \in w_{v}} y^{-1} \cdot \mathrm{SL}^{-1} \cdot \lambda$, a $W_{v}$-invariant subset of weights.

LEMMA. Let $y \in W_{v}$ and let $\alpha$ be a simple root such that $l\left(y_{\alpha} y\right)=l(y)$ +1 . If $\chi \in F^{v}$, then $y_{\alpha} y \cdot \chi \in \mathrm{SL}^{-1} \cdot(y \cdot \chi)$.

Proof. We may take $v=-\rho$. Since $l\left(s_{\alpha} w\right)=l(w)+1$, we have $w^{-1}(\alpha) \in R^{+}$. Hence, $s_{\alpha} w \cdot \chi-w \cdot \chi=-\left(\alpha^{\nu}, w \cdot \chi+\rho\right) \alpha=$ $-\left(w^{-1}(\alpha)^{\nu}, \chi+\rho\right) \alpha \geq 0$, when $\chi \in F^{-\rho}$.

In the same way, if $\chi \in F_{v}$, then $y_{\alpha} y \cdot \chi \in \mathrm{SL} \cdot(y \cdot \chi)$.

0.4. We take the following facts about infinitesimally induced modules from [5] and [2]. For $\lambda \in X(T)$, let $Z_{1}(\lambda)$ be the $T G_{1}$-module induced from the character $\lambda$ on $T B_{1} . Z_{1}(\lambda)$ is isomorphic, as a $T G_{1}$-module, with the $B G_{1}$-module induced from the character $\lambda$ on $B$. When we regard $Z_{1}(\lambda)$ as a $B G_{1}$-module in that way, we denote it by $B Z_{1}(\lambda) . M_{1, \lambda}$ denotes the unique simple submodule of $B Z_{1}(\lambda)$. The composition factors of $B Z_{1}(\lambda)$ lie in the set $\left\{M_{1, \chi} \mid \chi \in X(T)\right\}$. A composition series for $B Z_{1}(\lambda)$ is also a $\left(T G_{1^{-}}\right)$composition series for $Z_{1}(\lambda)$. To see that $M_{1, \chi}$ is irreducible as a $T G_{1}$-module, we have $B Z_{1}(\lambda)=B Z_{1}\left(\lambda^{0}\right) \otimes p \lambda^{1}$, and $M_{1, \lambda}=M_{1, \lambda^{0}} \otimes p \lambda^{1} . M_{1, \lambda^{0}}$ is isomorphic, as a $B G_{1}$-module, with the $G$-module $M_{\lambda^{0}}$, and $M_{\lambda^{0}}$ remains irreducible as a $G_{1}$-module, since $\lambda^{0} \in$ $V^{(p-1) \rho}$.

Let $h$ be the Coxeter number of $(G, T)$. Let $C_{1}$ be the alcove in $V_{-\rho}$ which contains $-\rho$ in its closure.

Definition [2]. $\lambda=\lambda^{0}+p \lambda^{1}$ is generic if $6(h-1) \leq\left(\alpha^{\nu}, \lambda^{1}\right) \leq p-$ $6(h-1)$, for all $\alpha \in R^{+}$.

The following information may be found in [2], for $\lambda$ generic. Let $\mu=\mu^{0}+p \mu^{1}$ be a weight.

1. $H^{i}(w \cdot \lambda)=0$ for $i \neq l(w)$

2. If $\left[B Z_{1}(w \cdot \lambda): M_{1, \mu}\right] \neq 0$, then $\mu^{1} \in w \cdot C_{1}$. 
3. If $\mu^{1} \in w \cdot C_{1}$, then $H^{l(w)}\left(G / B G_{1},\left(M_{1, \mu}\right)\right)=M_{\mu^{0}+p w^{-1} \cdot \mu^{1}}$.

4. $H^{l(w)}\left(G / B G_{1},-\right)$ takes a composition series for $B Z_{1}(w \cdot \lambda)$ to a $G$-composition series for $H^{l(w)}(w \cdot \lambda)$.

5. $\left[H^{l(w)}(w \cdot \lambda): M_{\mu}\right]=\left[B Z_{1}(w \cdot \lambda): M_{1, \mu^{0}+p w \cdot \mu^{1}}\right]$, for $\mu \in X(T)^{+}$.

1.

1.1. We require the following material from $[2,3]$ on connections between sheaf cohomology ( $G$-) modules and induced modules for infinitesimal subgroups of $G$, established by Cline, Parshall, Scott, Jantzen and Andersen.

For $\lambda$ and $w^{-1} \cdot(\lambda+p \theta)$ generic weights, we have the following equalities, by 0.4 .

$$
\begin{aligned}
{\left[H^{0}(\lambda): M_{\mu^{0}+p \mu^{1}}\right] } & =\left[B Z_{1}(\lambda): M_{1, \mu^{0}+p \mu^{1}}\right] \\
& =\left[B Z_{1}(\lambda) \otimes p \theta: M_{1, \mu^{0}+p \mu^{1}} \otimes p \theta\right] \\
& =\left[B Z_{1}(\lambda+p \theta): M_{1, \mu^{0}+p\left(\mu^{1}+\theta\right)}\right] \\
& =\left[H^{l(w)}(\lambda+p \theta): M_{\mu^{0}+p w^{-1} \cdot\left(\mu^{1}+\theta\right)}\right] \\
& =\left[H^{0}\left(w^{-1} \cdot(\lambda+p \theta)\right): M_{\mu^{0}+p w^{-1} \cdot\left(\mu^{1}+\theta\right)}\right] .
\end{aligned}
$$

In the following proposition, $p \theta$ is determined by requiring that $w^{-1} \cdot(\lambda+p \theta)=y^{-1} \cdot \lambda$.

Proposition. Let $\lambda$ and $y^{-1} \cdot \lambda$ be generic weights.

(a) $\left[H^{0}(\lambda): M_{\mu^{0}+p \mu^{1}}\right]=\left[H^{0}\left(y^{-1} \cdot \lambda\right): M_{\mu^{0}+p w^{-1}\left(\mu^{1}+\theta\right)}\right]$.

(b) $I_{y^{-1}} \cdot\left(\mu^{0}+p \mu^{1}\right)=\mu^{0}+p w^{-1} \cdot\left(\mu^{1}+\theta\right)$.

Proof. (a) is proved above.

(b) $\mu^{0}+p \mu^{1}$ lies in $V^{p \mu^{1}+(p-1) \rho}$. Since $I_{y^{-1}}$ operates on $V^{p \mu^{1}+(p-1) \rho}$ by

$$
T_{v^{-1} \cdot\left(p \mu^{1}+(p-1) \rho\right)-\left(p \mu^{1}+(p-1) \rho\right)}=T_{y^{-1}\left(p \mu^{1}+p \rho\right)-\left(p \mu^{1}+p \rho\right)},
$$

we have

$$
\begin{aligned}
I_{y^{-1}} \cdot\left(\mu^{0}+p \mu^{1}\right) & =\mu^{0}+p \mu^{1}+y^{-1}\left(p \mu^{1}+p \rho\right)-\left(p \mu^{1}+p \rho\right) \\
& =\mu^{0}-p \rho+y^{-1}\left(p \mu^{1}+p \rho\right) .
\end{aligned}
$$

Beginning with the other side of equation (b), we have

$$
\mu^{0}+p\left(w^{-1} \cdot\left(\mu^{1}+\theta\right)\right)=\mu^{0}+w^{-1}\left(p \mu^{1}+p \theta+p \rho\right)-p \rho .
$$

Hence, the equality (b) is equivalent to

$$
y^{-1}\left(p \mu^{1}+p \rho\right)=w^{-1}\left(p \mu^{1}+p \theta+p \rho\right) .
$$


The last equation is equivalent to

$$
w y^{-1} \cdot\left(p \mu^{1}+(p-1) \rho\right)-\left(p \mu^{1}+(p-1) \rho\right)=w y^{-1} \cdot \lambda-\lambda
$$

a valid equation since $w y^{-1} \cdot \lambda-\lambda$ is independent of $\lambda$. In fact, $w y^{-1}=$ $w T_{v+\rho} w^{-1} T_{-v-\rho}=T_{w(v+\rho)} T_{-v-\rho}$ is a translation operator.

As a corollary of the proposition, we get the alcove transition property of [7]. Let $I_{w_{v}}$ act on $X(T) \times \mathbf{N}$ by $I_{y} \cdot(\chi, n)=\left(I_{y} \cdot \chi, n\right)$.

COROLlaRY. $I_{y} \cdot m X_{\lambda}=m X_{y \cdot \lambda}$.

The cohomology modules $H^{0}(\lambda)$ and $H^{l(w)}\left(w y^{-1} \cdot \lambda\right)$ can be developed in stages, namely,

$$
H^{0}(\lambda)=H^{0}\left(G / B G_{1}, H^{0}\left(B G_{1} / B, L(\lambda)\right)\right)
$$

and

$$
H^{l(w)}\left(w y^{-1} \cdot \lambda\right)=H^{l(w)}\left(G / B G_{1}, H^{0}\left(B G_{1} / B, L\left(w y^{-1} \cdot \lambda\right)\right)\right) .
$$

In [3], Andersen shows that, for $\lambda$ generic,

$$
H^{0}\left(G / B G_{1}, \operatorname{Soc}^{j}\left(Z_{1}(\lambda)\right)\right)=\operatorname{Soc}^{j}\left(H^{0}(\lambda)\right)
$$

and

$$
H^{l(w)}\left(G / B G_{1}, \operatorname{Soc}^{J}\left(Z_{1}(w \cdot \lambda)\right)\right) \subset \operatorname{Soc}^{J}\left(H^{l(w)}(w \cdot \lambda)\right)
$$

In those statements, it is understood that $\operatorname{Soc}^{j}\left(Z_{1}(\lambda)\right)$ is the $j$ th $T G_{1}$-socle term, which is a $B G_{1}$-submodule of $B Z_{1}(\lambda)$, since $G_{1}$ is a normal subgroup of $B G_{1}$ and since the $G_{1}$-socle series of $Z_{1}(\lambda)$ coincides with the $T G_{1}$-socle series of $Z_{1}(\lambda)$.

1.2. Proof of the $W$-linkage conjecture. We prove the conjecture of [7], which states that the set $X_{\lambda}$ of highest weights of composition factors of $H^{0}(\lambda)$ equals the $W$-linkage class of $\lambda$, when $\lambda$ is a generic weight. At the suggestion of J. E. Humphreys, we use a Theorem of J.-c. Ye [9] which gives the composition factors of the infinitesimally induced $T G_{1}$-modules $Z_{1}(\lambda)$.

Let $\mathscr{S}_{1, \lambda}=\left\{\mu \in X(T)^{+} \mid \mu \uparrow\left(w_{0} \cdot \lambda+p \rho\right)\right\}$. Let $\lambda$ be a $p$-regular weight in $V^{(p-1) \rho}$.

THEOREM [Cor. 3.5 of [9]]. Suppose that $p>2 h-2$.

$$
\left\{\lambda^{\prime} \in X(T) \mid\left[Z_{1}\left(\lambda^{\prime}\right): M_{1, \lambda}\right] \neq 0\right\}=\left\{w \cdot \mu+p \rho \mid \mu \in \mathscr{S}_{1, \lambda}, w \in W\right\} .
$$


Since $Z_{1}\left(\lambda^{\prime}\right) \otimes(-p \rho)=Z_{1}\left(\lambda^{\prime}-p \rho\right)$, we get the following shifted version of the theorem, when we add $-p \rho$ to each weight in the sets of the Theorem, and replace $\lambda-p \rho$ by $\lambda$. 2.

THEOREM. Let $\lambda$ be a p-regular weight in $V^{-\rho}$. Suppose that $p>2 h-$

$$
\left\{\lambda^{\prime} \in X(T) \mid\left[Z_{1}\left(\lambda^{\prime}\right): M_{1, \lambda}\right] \neq 0\right\}=\left\{w \cdot \mu \mid \mu \in \mathscr{S}_{1, \lambda+p \rho}, w \in W\right\} .
$$

Proposition. If $\lambda \in V^{-\rho}$, then $W L^{-1} \cdot \lambda=\left\{w \cdot \mu \mid \mu \in \mathscr{S}_{1, \lambda+p \rho}\right.$, $w \in W\}$.

Proof. $\mathscr{S}_{1, \lambda+p \rho}=\left\{\mu \in X(T)^{+} \mid \mu \uparrow w_{0} \cdot \lambda\right\}$. Since $\lambda \in V^{-\rho}, W L^{-1} \cdot \lambda$ $=\bigcap_{w \in W} w \cdot\left(\mathrm{SL}^{-1} \cdot \lambda\right)$. Let $\mu \in \mathscr{S}_{1, \lambda+p \rho}$ and let $w \in W$. By the lemma in $0.3, w_{1} w \cdot \mu \uparrow w_{0} \cdot \lambda$ for each $w_{1} \in W$; hence, $\lambda \uparrow w_{0} w_{1} w \cdot \mu$. In other symbols, $w_{0} w_{1} w \cdot \mu \in \mathrm{SL}^{-1} \cdot \lambda$ and $w \cdot \mu \in \bigcap_{w_{1} \in W}\left(w_{0} w_{1}\right)^{-1} \cdot\left(\mathrm{SL}^{-1} \cdot \lambda\right)=$ $W L^{-1} \cdot \lambda$. To get the other inclusion, it will suffice to show that $F_{-\rho} \cap$ $W L^{-1} \cdot \lambda \subset \mathscr{S}_{1, \lambda+p \rho}$, since $W L^{-1} \cdot \lambda$ and $\left\{w \cdot \mu \mid \mu \in \mathscr{S}_{1, \lambda+p \rho}, w \in W\right\}$ are each $W$-stable. If $\chi \in F_{-\rho} \cap W L^{-1} \cdot \lambda$, then $\lambda \uparrow w \cdot \chi$ for all $w \in W$, and $w_{0} w \cdot \chi \uparrow w_{0} \cdot \lambda$. In particular, $\chi \uparrow w_{0} \cdot \lambda$.

Let $p>2 h-2$.

THEOREM (The $W$-linkage Theorem). Let $\lambda$ be a p-regular generic weight. The set of highest weights of composition factors of $H^{0}(\lambda)$ equals the $W$-linkage class of $\lambda$.

Proof. Let $X_{1}^{\lambda}=\left\{\lambda^{\prime} \in X(T) \mid\left[Z_{1}\left(\lambda^{\prime}\right): M_{1, \lambda}\right] \neq 0\right\}$ and $X_{1, \lambda}=\left\{\lambda^{\prime} \in\right.$ $\left.X(T) \mid\left[Z_{1}(\lambda): M_{1, \lambda^{\prime}}\right] \neq 0\right\}$, for any weight $\lambda$. By the proposition and theorem above, we have $W L^{-1} \cdot \lambda=X_{1}^{\lambda}$, for $p$-regular weights $\lambda$ in $V^{-\rho}$; hence, by translation by $v+\rho$, we have that equality for $p$-regular weights $\lambda$ in any box $V^{v}$. Equivalently, $W L \cdot \lambda=X_{1, \lambda}$ for $p$-regular weights. By $0.4(5), X_{\lambda}=X_{1, \lambda}=W L \cdot \lambda$, for $\lambda$ generic.

Definition. Let $\lambda$ be a generic weight. $X_{g}^{\lambda}$ is the set of generic weights in $X^{\lambda}$.

THEOREM. (The inverse $W$-linkage Theorem.) Let $\lambda$ be a p-regular generic weight. If all the weights in $W L^{-1} \cdot \lambda$ are generic, then $X_{g}^{\lambda}=$ $W L^{-1} \cdot \lambda$.

Proof. If $\mu \in X_{g}^{\lambda}$, then $\lambda \in X_{\mu}=W L \cdot \mu$, by the $W$-linkage Theorem. Hence, $\mu \in W L^{-1} \cdot \lambda$. Using the hypothesis, the argument may be reversed to give the reverse containment. 
1.3. Extensions, the second socle level, and alcove transition. For $M$ a $G$-module, set $X[M]=\left\{(\chi, n) \in X(T)^{+} \times \mathbf{N} \mid n=\left[M: M_{\chi}\right]\right\}$. In particular, $X\left[H^{0}(\lambda)\right]=m X_{\lambda}$. We will show that alcove transition stabilizes the set $m \operatorname{Ext}(\lambda)^{0}$, and preserves the second socle level, i.e.,

$$
I_{y^{-1}} \cdot X\left[\operatorname{Soc}^{2}\left(H^{0}(\lambda)\right)\right]=X\left[\operatorname{Soc}^{2}\left(H^{l(w)}\left(w y^{-1} \cdot \lambda\right)\right)\right] .
$$

By [7], $I_{y^{-1}} \cdot X\left[\operatorname{Soc}^{1}\left(H^{0}(\lambda)\right)\right]=X\left[\operatorname{Soc}^{1}\left(H^{l(w)}\left(w y^{-1} \cdot \lambda\right)\right)\right]$.

Define the usual ordering on the set of functions from $X(T)^{+}$to $\mathbf{N}$ by: $\left\{\left(\chi, n_{\chi}\right) \in X(T)^{+} \times \mathbf{N}\right\} \leq\left\{\left(\chi, m_{\chi}\right) \in X(T)^{+} \times \mathbf{N}\right\}$ if $n_{\chi} \leq m_{\chi}$, for each $\chi \in X(T)^{+}$. In the following proposition, we express a part of Andersen's results [3], quoted in 1.1, in terms of alcove transition.

Proposition. Let $\lambda$ and $y^{-1} \cdot \lambda$ be generic weights. For $j \geq 1$,

$$
I_{y^{-1}} \cdot X\left[\operatorname{Soc}^{j}\left(H^{0}(\lambda)\right)\right] \leq X\left[\operatorname{Soc}^{J}\left(H^{l(w)}\left(w y^{-1} \cdot \lambda\right)\right)\right] .
$$

Proof. Set $p \theta=w y^{-1} \cdot \lambda-\lambda$. We have

$$
\begin{aligned}
{\left[\operatorname{Soc}^{j}\left(H^{0}(\lambda)\right): M_{\mu^{0}+p \mu^{1}}\right] } & =\left[\operatorname{Soc}^{j}\left(Z_{1}(\lambda)\right): M_{1, \mu^{0}+p \mu^{1}}\right] \\
& =\left[\operatorname{Soc}^{j}\left(Z_{1}(\lambda+p \theta)\right): M_{1, \mu^{0}+p\left(\mu^{1}+\theta\right)}\right] \\
& \leq\left[\operatorname{Soc}^{j}\left(H^{l(w)}(\lambda+p \theta)\right): M_{\mu^{0}+p w^{-1} \cdot\left(\mu^{1}+\theta\right)}\right] .
\end{aligned}
$$

Since $I_{y^{-1}} \cdot\left(\mu^{0}+p \mu^{1}\right)=\mu^{0}+p w^{-1} \cdot\left(\mu^{1}+\theta\right)$, the proof is complete.

Lemma. Let $M$ be a module with irreducible socle $M_{\lambda}$. If $M / M_{\lambda} \cong$ $\oplus_{i=1}^{m} M_{\chi}$, where $\chi \neq \lambda$, then $\operatorname{dim}_{k} \operatorname{Ext}_{G}^{1}\left(M_{\chi}, M_{\lambda}\right) \geq m$.

Proof. The exactness of the sequence

$$
\begin{aligned}
0 & \rightarrow \operatorname{Hom}_{G}\left(M_{\chi}, M_{\lambda}\right) \rightarrow \operatorname{Hom}_{G}\left(M_{\chi}, M\right) \rightarrow \operatorname{Hom}_{G}\left(M_{\chi}, M / M_{\lambda}\right) \\
& \rightarrow \operatorname{Ext}_{G}^{1}\left(M_{\chi}, M_{\lambda}\right)
\end{aligned}
$$

and the hypothesis that $\operatorname{Hom}_{G}\left(M_{\chi}, M\right)=(0)$ imply the statement.

The following Theorem of Andersen's [2, Th. 3.6] shows that extension multiplicity is constant on alcove transition orbits.

TheOREM 1 [2]. Let $\lambda$ be a generic weight in $V^{v}$. For each $y \in W_{v}$,

$$
I_{y} \cdot m \operatorname{Ext}(\lambda)^{0}=m \operatorname{Ext}(\lambda)^{0} .
$$

REMARKs 1 . To reconcile the notation in Theorem 1 with the notation of $[2,3.6]$, one may use the formula

$$
I_{y, n} \cdot\left(\mu^{0}+p^{n}\left(\lambda^{1}+\mu^{1}\right)\right)=\mu^{0}+p^{n}\left(\lambda^{1}+w\left(\mu^{1}\right)\right),
$$

relative to the vertex $v=p^{n} \lambda^{1}+\left(p^{n}-1\right) \rho$. Here, $I_{y, 1}=I_{y}$. 
2. It is instructive to see how the Theorem follows from the previous proposition, given sufficient genericity assumptions. Let $\left(\delta, n_{\delta}\right)$ be an element of $m \operatorname{Ext}(\lambda)^{0}$, where $n_{\delta}$ may be zero. Assume that all the weights in $W_{v} \cdot I_{w_{v}} \cdot \delta$ are generic. To prove the Theorem, we show that $n_{\delta}=n_{\mu}$, for each $\mu \in I_{w_{v}} \cdot \delta$. Let $\chi$ be an element of $I_{w_{v}} \cdot \delta$ which maximizes the extension multiplicity relative to $\lambda$, i.e.,

$$
n_{\chi}=\max \left\{n_{\mu} \mid \mu \in I_{w_{v}} \cdot \delta,\left(\mu, n_{\mu}\right) \in m \operatorname{Ext}(\lambda)^{0}\right\} .
$$

Let $y \in W_{v}$.

(i) If $\chi<\lambda$, then $\left(\chi, n_{\chi}\right) \in X\left[\operatorname{Soc}^{2}\left(H^{0}(\lambda)\right)\right]$, by [4]. Hence,

$$
\begin{aligned}
I_{y} \cdot\left(\chi, n_{\chi}\right) & =\left(I_{y} \cdot \chi, n_{\chi}\right) \in I_{y} \cdot X\left[\operatorname{Soc}^{2}\left(H^{0}(\lambda)\right)\right] \\
& \leq X\left[\operatorname{Soc}^{2}\left(H^{l\left(w^{-1}\right)}\left(w^{-1} y \cdot \lambda\right)\right)\right],
\end{aligned}
$$

by the proposition above. Since $M_{\lambda}$ is the socle of $H^{l\left(w^{-1}\right)}\left(w^{-1} y \cdot \lambda\right)$, the lemma above implies that the extension multiplicity $n_{I_{y} \cdot \chi}$ of $I_{y} \cdot \chi$ relative to $\lambda$ is at least $n_{\chi}$. By the maximality of $n_{\chi}$, we have $n_{\chi}=n_{I_{\nu} \cdot \chi}$. have

(ii) If $\chi>\lambda$, then $\left(\lambda, n_{\chi}\right) \in X\left[\operatorname{Soc}^{2}\left(H^{0}(\chi)\right)\right]$. Hence, for $\lambda \in V^{v}$, we

$$
\begin{aligned}
\left(\lambda, n_{\chi}\right) & =I_{y} \cdot\left(\lambda, n_{\chi}\right) \in I_{y} \cdot X\left[\operatorname{Soc}^{2}\left(H^{0}(\chi)\right)\right] \\
& \leq X\left[\operatorname{Soc}^{2}\left(H^{l\left(w^{-1}\right)}\left(w^{-1} y \cdot \chi\right)\right)\right],
\end{aligned}
$$

by the genericity assumptions. Since $\operatorname{Soc}^{1}\left(H^{l\left(w^{-1}\right)}\left(w^{-1} \cdot y \cdot \chi\right)\right)=M_{I_{y} \cdot \chi}$, it follows that $n_{\chi} \leq n_{I_{y} \cdot \chi}$, as in (i). Hence, $n_{\chi}=n_{I_{v} \cdot \chi}$.

The following is a Corollary to [2, Th. 3.6].

Corollary. If $\lambda=\lambda^{0}+p \lambda^{1}$ satisfies $8(h-1) \leq\left(\alpha^{v}, \lambda^{1}\right) \leq p-$ $8(h-1)$ for all $\alpha \in R^{+}$, then all the weights in $\operatorname{Ext}(\lambda)^{0}$ are generic.

Proof. Let $\delta$ be an element of $\operatorname{Ext}(\lambda)^{0}$. If we write

$$
\delta=\mu^{0}+p\left(\lambda^{1}+w\left(\mu^{1}\right)\right)
$$

for some $\mu \in X(T)^{+}$, then we have $\left|\left(\alpha^{\nu}, \mu^{1}\right)\right|<2(h-1)$, by Lemma 3.5 and Theorem 3.6 of [2]. Hence, $6(h-1)<\left(\alpha^{\nu}, \lambda^{1}+\mu^{1}\right)<p-6(h-1)$.

We have the following conjecture on the transformation properties of alcove transition. Let $y \in W_{v}$.

Conjecture. If $\chi$ and $y \cdot \chi$ are generic weights, then $I_{y}$. $X\left[\operatorname{Soc}^{j}\left(H^{l(w)}(w \cdot \chi)\right)\right]=X\left[\operatorname{Soc}^{j}\left(H^{0}(y \cdot \chi)\right)\right]$.

The conjecture is valid for the second socle term. 
THEOREM 2. If $\lambda$ and $y^{-1} \cdot \lambda$ are generic weights, where $\lambda \in V^{v}$, then

$$
I_{y^{-1}} \cdot X\left[\operatorname{Soc}^{2}\left(H^{0}(\lambda)\right)\right]=X\left[\operatorname{Soc}^{2}\left(H^{l(w)}\left(w y^{-1} \cdot \lambda\right)\right)\right] .
$$

The statements of two corollaries precede the proof. Let $M$ be a $G$-module. Let $M \cap m \operatorname{Ext}(\lambda)$ denote the set $\left\{\left(\chi, n_{\chi}\right) \in m \operatorname{Ext}(\lambda) \mid\right.$ $\left.\left[M: M_{\chi}\right] \neq 0\right\}$, i.e., the highest weights $\chi$ of the composition factors of $M$, paired with the integer $n_{\chi}=\operatorname{dim}_{k} \operatorname{Ext}_{G}^{1}\left(M_{\chi}, M_{\lambda}\right)$. The socle of $H^{(l(w)}\left(w y^{-1} \cdot \lambda\right)$ is $M_{\lambda}$, since $y^{-1} \cdot \lambda$ is a generic weight (see [2] and [7]). Let $\operatorname{Soc}_{2}=\operatorname{Soc}^{2} / \operatorname{Soc}^{1}$.

We use implicitly in the arguments below the fact that $\operatorname{Ext}(\lambda) \cap \mathrm{SL}$. $\lambda=\operatorname{Ext}(\lambda)^{0} \cap \mathrm{SL} \cdot \lambda$, for $\lambda p$-regular. To see that fact, we have $\operatorname{Ext}_{G}^{1}\left(M_{\chi^{0}+p \chi^{1}}, M_{\lambda^{0}+p \lambda^{1}}\right) \cong \operatorname{Ext}_{G}^{1}\left(M_{\chi^{1}}, M_{\lambda^{1}}\right)$ whenever $\chi^{0}=\lambda^{0}$ and $\lambda$ is not on a wall of the dominant chamber [10]. Since $\lambda^{1} \in C_{0}$, we conclude that if $\operatorname{Ext}_{G}^{1}\left(M_{\chi^{1}}, M_{\lambda^{1}}\right) \neq 0$, then $\chi^{1}>\lambda^{1}$, by linkage. Hence $\chi^{0}+p \chi^{1}>$ $\lambda^{0}+p \lambda^{1}$.

Corollary 1.

$$
H^{l(w)}\left(w y^{-1} \cdot \lambda\right) \cap m \operatorname{Ext}(\lambda)=X\left[\operatorname{Soc}_{2}\left(H^{l(w)}\left(w y^{-1} \cdot \lambda\right)\right)\right] .
$$

Consequently, $\operatorname{Soc}^{2}\left(H^{l(w)}\left(w y^{-1} \cdot \lambda\right)\right)$ realizes all extensions of $M_{\chi}$ by $M_{\lambda}$, whenever $M_{\chi}$ is a composition factor of $H^{l(w)}\left(w y^{-1} \cdot \lambda\right)$.

COROllary 2.

$$
H^{l(w)}\left(G / B G_{1}, \operatorname{Soc}^{2}\left(Z_{1}\left(w y^{-1} \cdot \lambda\right)\right)\right)=\operatorname{Soc}^{2}\left(H^{l(w)}\left(w y^{-1} \cdot \lambda\right)\right) .
$$

Proof of the Theorem. Let $\left(\chi, m_{\chi}\right) \in X\left[\operatorname{Soc}_{2}\left(H^{l(w)}\left(w y^{-1} \cdot \lambda\right)\right)\right]$ and let $\left(I_{y} \cdot \chi, m_{I_{v} \cdot \chi}\right) \in X\left[\operatorname{Soc}_{2}\left(H^{0}(\lambda)\right)\right]$. We need to show that $m_{\chi}=m_{I_{y} \cdot \chi}$. By 1.3, proposition, $m_{I_{v} \cdot \chi} \leq m_{\chi} \cdot m_{I_{y} \cdot \chi}$ is also equal to $\operatorname{dim}_{k} \operatorname{Ext}_{G}^{1}\left(M_{\lambda}, M_{I_{y} \cdot \chi}\right)$ [4], and $m_{\chi}$ is less than or equal to $\operatorname{dim}_{k} \operatorname{Ext}_{G}^{1}\left(M_{\lambda}, M_{\chi}\right)$. Hence, by Theorem $1, m_{\chi} \leq m_{I_{v} \cdot \chi}$.

Proof of Corollary 1. $I_{y^{-1}}$ transforms the equality $H^{0}(\lambda) \cap m \operatorname{Ext}(\lambda)$ $=X\left[\operatorname{Soc}_{2}\left(H^{0}(\lambda)\right)\right]$ into the equality of the Corollary.

Proof of Corollary 2. Since $H^{l(w)}\left(G / B G_{1}, \operatorname{Soc}^{2}\left(Z_{1}\left(w y^{-1} \cdot \lambda\right)\right)\right) \subset$ $\operatorname{Soc}^{2}\left(H^{l(w)}\left(w y^{-1} \cdot \lambda\right)\right)$ by 1.1 , it suffices to show that the two modules have the same number of composition factors. Let $n(M)$ be the length of a composition series for a module $M$. 
Since $\lambda$ and $w y^{-1} \cdot \lambda$ are translates of each other, we have, using $0.4(4)$,

$$
\begin{aligned}
n\left(H ^ { l ( w ) } \left(G / B G_{1},\right.\right. & \left.\left.\operatorname{Soc}^{2}\left(Z_{1}\left(w y^{-1} \cdot \lambda\right)\right)\right)\right)=n\left(\operatorname{Soc}^{2}\left(Z_{1}\left(w y^{-1} \cdot \lambda\right)\right)\right) \\
= & n\left(\operatorname{Soc}^{2}\left(Z_{1}(\lambda)\right)\right)=n\left(H^{0}\left(G / B G_{1}, \operatorname{Soc}^{2}\left(Z_{1}(\lambda)\right)\right)\right) .
\end{aligned}
$$

By 1.1 and the Theorem,

$$
\begin{aligned}
n\left(H^{0}\left(G / B G_{1}, \operatorname{Soc}^{2}\left(Z_{1}(\lambda)\right)\right)\right) & =n\left(\operatorname{Soc}^{2}\left(H^{0}(\lambda)\right)\right) \\
& =n\left(\operatorname{Soc}^{2}\left(H^{l(w)}\left(w y^{-1} \cdot \lambda\right)\right)\right) .
\end{aligned}
$$

2. Estimates for $\operatorname{Ext}(\lambda)^{0}$. Throughout this section, all the weights considered are $p$-regular.

2.1. We begin with two lemmas on the geometry of the orbits for the operation of the alcove transition group on $X(T)$. In the first lemma, we show that each orbit for $I_{W_{v}}$ operating on the space of $p$-regular weights contains unique maximal and minimal weights relative to the strong linkage ordering $\uparrow$.

Let $v$ be the special vertex $\bigcap_{\beta \in S, n_{\beta} \in \mathbf{Z}} H_{\beta, n_{\beta} p}$ and let $v_{-1}=$ $\bigcap_{\beta \in S, n_{\beta} \in \mathbf{Z}} H_{\beta,\left(n_{\beta}-1\right) p}$. Let the orbit of a weight $\chi$ be denoted by $I \cdot \chi$.

LEMMA 1. There are unique elements $\delta$ and $\delta^{\prime}$ in $I \cdot \chi$ such that $I \cdot \chi \subset \mathrm{SL} \cdot \delta$ and $I \cdot \chi \subset \mathrm{SL}^{-1} \cdot \delta^{\prime}$. Furthermore, $\delta$ and $\delta^{\prime}$ are the only elements of $I \cdot \chi$ lying in $F_{v_{-1}}$ and $F^{v}$, respectively.

Proof. It is evident that there can be no more than one such element $\delta$ and one such element $\delta^{\prime}$. Let $\bar{v}$ be the vertex such that $\chi \in V^{\bar{v}}$, and let $\bar{y}$ be an element of $W_{v}$ such that $\bar{y} \cdot \bar{v} \in F_{v}$. We claim that $\delta=I_{\bar{y}} \cdot \chi$ satisfies SL $\cdot \delta \supset I \cdot \chi$. In fact, we show that if $l\left(y_{\alpha} y\right)=l(y)+1$, then $I_{y_{\alpha}} I_{y} \cdot \delta \in \mathrm{SL} \cdot\left(I_{y} \cdot \delta\right)$. By the lemma in 0.3 for $F_{v}$, if $l\left(y_{\alpha} y\right)=l(y)+1$ and $\mu \in F_{v}$, then $y_{\alpha} y \cdot \mu \in \mathrm{SL} \cdot(y \cdot \mu)$. Since $\delta=I_{\bar{y}} \cdot \chi \in V^{\bar{y} \cdot \bar{v}}$ and $I_{y} \cdot$ $\delta \in V^{y \bar{y} \cdot \bar{v}}$, we have $I_{y_{\alpha}} I_{y} \cdot \delta=I_{y} \cdot \delta+y_{\alpha} y \bar{y} \cdot \bar{v}-y \bar{y} \cdot \bar{v}$, where $y_{\alpha} y \bar{y} \cdot \bar{v}-$ $y \bar{y} \cdot \bar{v}=m p \alpha \geq 0$, by that lemma for $\mu=\bar{y} \cdot \bar{v}$. Hence, $I_{y_{\alpha}} I_{y} \cdot \delta \in \mathrm{SL}$. $\left(I_{y} \cdot \delta\right)$.

To complete the proof of the lemma as far as it concerns $\delta$, we show that $F_{v_{-1}} \cap I \cdot \chi=\{\delta\}$. Let $n p<\left(\beta^{\nu}, \delta+\rho\right)<(n+1) p$. By [7, 4.2], $\delta-I_{y_{\beta}} \cdot \delta=\left(n+1-n_{\beta}\right) p \beta$. Since $I_{y_{\beta}} \cdot \delta \in \mathrm{SL} \cdot \delta$, we have $n \geq n_{\beta}-1$, i.e. $\delta \in F_{v_{-1}}$. Let $\bar{\delta}$ be any element of $F_{v_{-1}} \cap I \cdot \chi$ and let $v^{\prime}$ be the vertex such that $\bar{\delta} \in V^{v^{\prime}}$. We have $\left(\beta^{\nu}, \bar{\delta}+\rho\right)>\left(n_{\beta}-1\right) p$, and $\left(\beta^{v}, v^{\prime}+\rho\right) \geq n_{\beta} p$ and $v^{\prime} \in F_{v}$. A repetition of the argument given in the 
first part of the proof, with $\delta$ replaced by $\bar{\delta}$ and $\bar{y} \cdot \bar{v}$ by $v^{\prime}$, shows that $I \cdot \chi \subset \mathrm{SL} \cdot \bar{\delta}$. Hence, $\bar{\delta}=\delta$.

The facts about $\delta^{\prime}$ are proved in the same way as those for $\delta$, where $F^{v}$ replaces $F_{v}$ in the arguments.

In the second lemma, we show that $I_{w_{v}}$-orbits which lie totally within SL $\cdot \lambda$ also lie totally within $W L \cdot \lambda$. Let $\lambda$ be a $p$-regular weight in $V^{v}$.

Lemma 2. (a) $\bigcap_{y \in w_{v}} I_{y} \cdot(W L \cdot \lambda)=\bigcap_{y \in w_{v}} I_{y} \cdot(\mathrm{SL} \cdot \lambda)$.

(b) $\bigcap_{y \in W_{v}} I_{y} \cdot\left(W L^{-1} \cdot \lambda\right) \subset \bigcap_{y \in W_{v}} I_{y} \cdot\left(\mathrm{SL}^{-1} \cdot \lambda\right)$.

Proof. The left to right containments are evident. We prove the other containment in (a). Since $\lambda \in V^{v} \subset F^{v}$, we have $\mathrm{SL} \cdot \lambda \subset \mathrm{SL} \cdot\left(y^{-1} \cdot \lambda\right)$ for $y \in W_{v}$, by the lemma in 0.3. Hence

$$
\bigcap_{y \in W_{v}} I_{y} \cdot(\mathrm{SL} \cdot \lambda) \subset \bigcap_{y \in W_{v}} I_{y} \cdot \mathrm{SL} \cdot\left(y^{-1} \cdot \lambda\right)=W L \cdot \lambda,
$$

and

$$
\bigcap_{y \in W_{v}} I_{v} \cdot(\mathrm{SL} \cdot \lambda) \subset \bigcap_{y \in W_{v}} I_{y} \cdot(W L \cdot \lambda)
$$

2.2. Let $\lambda=\lambda^{0}+p \lambda^{1}$ be an element of $V^{v}$ satisfying the condition $8(h-1) \leq\left(\alpha^{\nu}, \lambda^{1}\right) \leq p-8(h-1)$, for all $\alpha \in R^{+}$. The operation of $I_{w_{v}}$ on $X(T)$ stabilizes $\operatorname{Ext}(\lambda)^{0}\left(1.3\right.$, Theorem 1), and the $I_{w_{v}}$-orbits in $\operatorname{Ext}(\lambda)$ may be divided into three types,

(i) the orbits within $W L \cdot \lambda$

(ii) the orbits within $W L^{-1} \cdot \lambda$

(iii) the orbits which meet both $W L \cdot \lambda$ and $W L^{-1} \cdot \lambda$.

In fact, $\operatorname{Ext}(\lambda)^{0} \subset X_{\lambda} \cup X_{g}^{\lambda}$, by [4] and the Corollary to Theorem 1 of 1.3; and $X_{\lambda} \cup X_{g}^{\lambda} \subset W L \cdot \lambda \cup W L^{-1} \cdot \lambda$, by the $W$-linkage Theorem.

Proposition 1. Let $\lambda \in V^{v}$ and let $\chi$ be a weight other than $\lambda$. If the orbit $I_{W_{v}} \cdot \chi$ lies in $W L \cdot \lambda \cup W L^{-1} \cdot \lambda$ and it intersects both $W L \cdot \lambda$ and $W L^{-1} \cdot \lambda$, then it equals $I_{W_{v}} \cdot s_{\beta *} \cdot \lambda$, for some $\beta \in S$.

Proof. Let $\chi$ be an element of the orbit. By the hypothesis, the orbit contains some element $\delta$ such that $\delta \in W L^{-1} \cdot \lambda$ and $I_{y_{\beta}} \cdot \delta \in W L \cdot \lambda$, for some $\beta \in S$. We will show that $I_{y_{\beta}} \cdot \delta=s_{\beta *} \cdot \lambda$.

First, we show that $I_{y_{\beta}} \cdot \delta=s_{\beta *}^{j *} \cdot \lambda$ for some $j \geq 1$. Since $I_{y_{\beta}} \cdot \delta \in \mathrm{SL}$ $\cdot \lambda$, and $\lambda \in \mathrm{SL}^{-1} \cdot \delta$, we have $0<\lambda-I_{y_{\beta}} \cdot \delta=\left(\delta-I_{y_{\beta}} \cdot \delta\right)+(\lambda-\delta)$ $=m p \beta-\sum_{\gamma \in R^{+}} a_{\gamma} \gamma$, where $m \in \mathbf{Z}$ and $a_{\gamma} \geq 0$. Hence, $a_{\gamma}=0$, for $\gamma \neq \beta$. Since $I_{y_{\beta}} \cdot \delta \in \mathrm{SL} \cdot \lambda$ and $I_{y_{\beta}} \cdot \delta=\lambda-m p \beta+a_{\beta} \beta$, we conclude that $I_{y_{\beta}} \cdot \delta=s_{\beta *}^{j} \cdot \lambda$ for some $j \geq 1$. 
The stability of $W L^{-1} \cdot \lambda$ under $W_{v}(0.3)$ implies that $y_{\beta} \cdot \delta \in W L^{-1}$. $\lambda$. Since $y_{\beta} \cdot \delta=y_{\beta} \cdot I_{y_{\beta}} \cdot s_{\beta *}^{j} \cdot \lambda=y_{\beta}^{2} s_{\beta}^{*} s_{\beta *}^{j} \cdot \lambda=s_{\beta *}^{j-1} \cdot \lambda, j$ must equal 1 .

Denote $I_{W_{v}} \cdot \chi$ by $I \cdot \chi$

Proposition 2. Let $\lambda$ be a weight in $V^{v}$.

(a) $\bigcap_{y \in W_{v}} I_{y} \cdot W L \cdot \lambda=\bigcup_{\delta \in F_{v-1} \cap \mathrm{SL} \cdot \lambda} I \cdot \delta$.

(b) $\bigcap_{y \in W_{v}} I_{y} \cdot W L^{-1} \cdot \lambda \subset \bigcup_{\delta \in F^{v} \cap \mathrm{SL}^{-1} \cdot \lambda} I \cdot \delta$.

Proof. By Lemma 2 of 2.1, it will suffice to show that $\bigcap_{y \in w_{v}} I_{y} \cdot \mathrm{SL}$. $\lambda=\bigcup_{\delta \in F_{v-1} \cap \mathrm{SL} \cdot \lambda} I \cdot \delta$ and $\bigcap_{y \in W_{v}} I_{y} \cdot \mathrm{SL}^{-1} \cdot \lambda=\bigcup_{\delta \in F^{v} \cap \mathrm{SL}^{-1} \cdot \lambda} I \cdot \delta$.

(i) Let $\delta \in F_{v_{-1}} \cap \mathrm{SL} \cdot \lambda$. By Lemma 1 of $2.1, I \cdot \delta \subset \mathrm{SL} \cdot \delta \subset \mathrm{SL}$. $\lambda$; hence, $I \cdot \delta \subset \cap_{y \in W_{v}} I_{y} \cdot \mathrm{SL} \cdot \lambda$.

(ii) Let $\chi \in \bigcap_{y \in w_{v}} I_{y} \cdot \mathrm{SL} \cdot \lambda$. Since $I \cdot \chi \subset \mathrm{SL} \cdot \lambda$, the element $\delta$ in the orbit, produced in Lemma 1 of 2.1, lies in $F_{v_{-1}} \cap \mathrm{SL} \cdot \lambda$. The proof of the second equality is similar.

As a consequence of Propositions 1 and 2, we have the following upper bound on $\operatorname{Ext}(\lambda)^{0}$.

THEOREM. Let $\lambda$ be a weight in $V^{v}$ satisfying the condition $8(h-1) \leq$ $\left(\alpha^{v}, \lambda^{1}\right) \leq p-8(h-1)$, for all $\alpha \in R^{+} . \operatorname{Ext}(\lambda)^{0}$ is contained in the union of the $I_{W_{-1}}$-orbits of the sets (i) $\left\{s_{\beta *} \cdot \lambda \mid \beta \in S\right\}$, (ii) $F_{v_{-1}} \cap \mathrm{SL} \cdot \lambda$, (iii) $F^{v} \cap \mathrm{SL}^{-1} \cdot \lambda$.

Here, the orbits of the elements of $F_{v_{-1}} \cap \mathrm{SL} \cdot \lambda$ lie in $W L \cdot \lambda$ and those of $F^{v} \cap \mathrm{SL}^{-1} \cdot \lambda$ lie in $W L^{-1} \cdot \lambda$.

EXAMPLES 1. For groups of type $A_{2}$ and $B_{2},\left(F_{v_{-1}} \cap \mathrm{SL} \cdot \lambda\right) \cup$ $\left(F^{v} \cap \mathrm{SL}^{-1} \cdot \lambda\right)=V^{v}$. Since $I_{W_{v}}$ operates trivially on $V^{v}$, we have that $\operatorname{Ext}(\lambda)^{0} \subset V^{v} \cup \bigcup_{\beta \in S} I_{W_{v}} \cdot s_{\beta *} \cdot \lambda$.

2. For the group of type $G_{2},\left(F_{v_{-1}} \cap \mathrm{SL} \cdot \lambda\right) \cup\left(F^{v} \cap \mathrm{SL}^{-1} \cdot \lambda\right)$ can exceed $V^{v}$. If $\lambda \in V^{v}$ lies in the alcove which contains the special vertex $v_{-1}$ in its closure, then $F^{v} \cap \mathrm{SL}^{-1} \cdot \lambda$ contains four weights outside $V^{v}$. Two of those weights belong to $\operatorname{Ext}(\lambda)^{0}$. If $\lambda \in V^{v}$ lies in the alcove which contains $v$ in its closure, then $F_{v_{-1}} \cap \mathrm{SL} \cdot \lambda$ contains four weights outside $V^{v}$. Again, two of those weights belong to $\operatorname{Ext}(\lambda)^{0}$. This shows the added complexity of the alcove geometry of $G_{2}$ over that of $A_{2}$ and $B_{2}$ for the study of extensions.

2.3. The first proposition below gives grounds for believing that $s_{\beta *} \cdot \lambda \in \operatorname{Ext}(\lambda)$, for $\beta \in S$. See 2.4 as well.

Let $\lambda$ be a generic weight. $M_{J_{w} \cdot \lambda}$ is the socle of $H^{l(w)}(w \cdot \lambda)$ and $\left[H^{l(w)}(w \cdot \lambda): M_{J_{w} \cdot \lambda}\right]=1$. See [7] and [2]. 
Lemma. $H^{0}(\lambda)$ has exactly $N+1$ socle levels $\Leftrightarrow M_{J_{w} \cdot \lambda}$ has socle level $l(w)+1$ in $H^{0}(\lambda)$ for all $w \in W$.

Proof. The number of socle levels of $H^{0}(\lambda)$ equals the socle level of its top factor $M_{J_{w_{0}} \cdot \lambda}$. That gives the right to left implication. Now suppose that $M_{J_{w_{0}} \lambda}$ is at the $l\left(w_{0}\right)+1$ socle level. To show that $M_{J_{w} \cdot \lambda}$ is at the $l(w)+1$-socle level, it suffices to show that the level of $M_{J_{\beta_{\beta}} w \cdot \lambda}$ exceeds that of $M_{J_{w} \cdot \lambda}$, whenever $l\left(s_{\beta} \cdot w\right)=l(w)+1$.

$M_{J_{w} \cdot \lambda}\left(\right.$ resp. $\left.M_{J_{s p} \cdot \lambda}\right)$ is the top factor of $H^{l\left(w_{0} w\right)}\left(w_{0} w \cdot \lambda\right)$ (resp. $\left.H^{I\left(n_{0} s_{\beta} w\right)}\left(w_{0} s_{\beta} \cdot w \cdot \lambda\right)\right)$ [7]. There is the part of the loop from [7]:

$$
H^{l\left(w_{0} w\right)}\left(w_{0} w \cdot \lambda\right) \rightarrow H^{l\left(w_{0} s_{\beta} \cdot w\right)}\left(w_{0} s_{\beta} w \cdot \lambda\right) \rightarrow \cdots \rightarrow H^{0}(\lambda) .
$$

Let $\psi$ be the (non-zero) composite of those mappings and let $\varphi$ be the composite of the mappings between $H^{l\left(w_{0} s_{\beta} w\right)}\left(w_{0} s_{\beta} w \cdot \lambda\right)$ and $H^{0}(\lambda)$. Since $\psi \neq 0, M_{J_{w} \cdot \lambda}$ and $M_{J_{S w^{w}} \cdot \lambda}$ are both factors of $\operatorname{Im}(\varphi)$, where $M_{J_{s w^{*}} \cdot \lambda}$ is the top factor of $\operatorname{Im}(\varphi)$. That completes the proof.

Proposition 1. If $H^{0}(\lambda)$ has exactly $N+1$ socle levels, then $\left(s_{\beta *} \cdot \lambda, 1\right) \in m \operatorname{Ext}(\lambda)$.

Proof. Since $M_{s_{\beta *} \cdot \lambda}=M_{J_{s_{\beta} \cdot \lambda}}$ has multiplicity one in $H^{0}(\lambda)$, we need only show that $s_{\beta *} \cdot \lambda \in \operatorname{Ext}\left(\lambda^{\beta}\right)$. By the lemma, $M_{J_{s_{\beta}} \cdot \lambda}$ has socle level 2 in $H^{0}(\lambda)$.

Proposition 2. $s_{\beta *} \cdot \lambda \in \operatorname{Ext}(\lambda) \Leftrightarrow$ the non-zero mapping $\psi$ : $H^{N-1}\left(w_{0} s_{\beta} \cdot \lambda\right) \rightarrow H^{0}(\lambda)$ has only the two factors $M_{\lambda}$ and $M_{s_{\beta *} \cdot \lambda}$ in its image.

Proof. The implication from right to left is evident. For the other implication, since $M_{s_{\beta *} \cdot \lambda}$ is the top factor of $H^{N-1}\left(w_{0} s_{\beta} \cdot \lambda\right)$, it is the top factor of $\operatorname{Im} \psi$. By the hypothesis, $M_{s_{\beta *} \cdot \lambda}$ is a factor of $\operatorname{Soc}^{2}\left(H^{0}(\lambda)\right)$, and so a factor of $\operatorname{Soc}^{2}(\operatorname{Im}(\psi))$.

EXAMPLE. For groups of type $A_{2}$ and $B_{2}$, the image of $\psi$ has just the two factors $M_{\lambda}$ and $M_{s_{\beta *} \cdot \lambda}$, by a computation in [7]; hence, $s_{\beta *} \cdot \lambda \in$ $\operatorname{Ext}(\lambda)$. Taking Example 1 of 2.2 into account, in order to settle the extension picture for $A_{2}$ and $B_{2}$, one need only find $V^{v} \cap \operatorname{Ext}(\lambda)$.

2.4. Let $\lambda$ be a weight in $V^{v}$ satisfying the condition $8(h-1) \leq$ $\left(\alpha^{v}, \lambda^{1}\right) \leq p-8(h-1)$, for all $\alpha \in R^{+}$. Let $C_{v_{-1}}$ be the alcove in $V^{v}$ which has $v_{-1}$ in its closure. For $\beta \in S$, we show that $s_{\beta^{*}} \cdot \lambda \in \operatorname{Ext}(\lambda)$ when $\lambda \in C_{v_{-1}}$. 
Let

$$
\begin{aligned}
v & =\bigcap_{\alpha \in S} H_{\alpha, n_{\alpha} p}, \\
v_{-1} & =\bigcap_{\alpha \in S} H_{\alpha,\left(n_{\alpha}-1\right) p}, \quad \text { and } \\
v^{\prime} & =H_{\beta,\left(n_{\beta}-1\right) p} \cap \bigcap_{\substack{\alpha \in S \\
\alpha \neq \beta}} H_{\alpha, n_{\alpha} p},
\end{aligned}
$$

for some fixed $\beta \in S$.

LEMma. If $\lambda \in C_{v_{-1}}$, then $s_{\beta *} \cdot \lambda \in V^{v^{\prime}}$.

Proof. We may assume that $v_{-1}=-\rho$. In that case, $v=(p-1) \rho=$ $\bigcap_{\alpha \in S} H_{\alpha, p}, s_{\beta *} \cdot \lambda=s_{\beta} \cdot \lambda$, and $0<\left(\gamma^{\nu}, \lambda+\rho\right)<p$, for each $\gamma \in R^{+}$. Since $s_{\beta}(\alpha) \in R^{+}$when $\alpha \in S$ and $\alpha \neq \beta$, we have that $\left(\alpha^{\nu}, s_{\beta} \cdot \lambda+\rho\right)$ $=\left(s_{\beta}(\alpha)^{\nu}, \lambda+\rho\right)$ lies between 0 and $p \cdot\left(\beta^{\nu}, s_{\beta} \cdot \lambda+\rho\right)=\left(s_{\beta}(\beta)^{\nu}, \lambda+\right.$ $\rho)=-\left(\beta^{\nu}, \lambda+\rho\right)$ lies between $-p$ and 0 . $S\}$.

Proposition 1. If $\lambda \in C_{v_{-1}}$, then $\operatorname{Ext}(\lambda) \cap \mathrm{SL} \cdot \lambda \subset\left\{s_{\beta *} \cdot \lambda \mid \beta \in\right.$

Proof. By the Theorem of 2.2, it suffices to show that $F_{v_{-1}} \cap \mathrm{SL} \cdot \lambda=$ $\{\lambda\}$ (a one element orbit) and that

$$
\mathrm{SL} \cdot \lambda \cap I_{W_{v}} s_{\beta *} \cdot \lambda=\left\{s_{\beta *} \cdot \lambda\right\} .
$$

The first of the two equalities is evident. To get the second equality, we have that $I_{W_{v}} s_{\beta *} \cdot \lambda \subset \mathrm{SL}^{-1} \cdot\left(s_{\beta *} \cdot \lambda\right)$, by Lemma 1 of 2.1 , since $s_{\beta *} \cdot \lambda$ $\in V^{v^{\prime}} \subset F^{v}$. Since SL $\cdot \lambda \cap \mathrm{SL}^{-1} \cdot\left(s_{\beta *} \cdot \lambda\right)=\left\{\lambda, s_{\beta *} \cdot \lambda\right\}$ and since $\{\lambda\}$ is a one element orbit, we have SL $\cdot \lambda \cap I_{W_{v}} \cdot s_{\beta *} \cdot \lambda=\left\{s_{\beta *} \cdot \lambda\right\}$.

Proposition 2. If $\lambda \in C_{v_{-1}}$, then $\operatorname{Soc}^{2}\left(H^{0}(\lambda)\right) / \operatorname{Soc}^{1}\left(H^{0}(\lambda)\right) \cong$ $\bigoplus_{\beta \in S} M_{s_{\beta *} \cdot \lambda}$.

Proof. Since each $M_{s_{\beta *} \cdot \lambda}$ has multiplicity one in $H^{0}(\lambda)$, Proposition 1 implies that

$$
\operatorname{Soc}^{2}\left(H^{0}(\lambda)\right) / \operatorname{Soc}^{1}\left(H^{0}(\lambda)\right) \cong \bigoplus_{\beta \in S_{1}} M_{s_{\beta} \cdot \lambda}
$$

for some subset $S_{1}$ of $S$. For each $\beta \in S$, consider a non-zero intertwining $\varphi_{\beta}: H^{0}(\lambda) \rightarrow H^{0}\left(s_{\beta *} \cdot \lambda\right)[\mathbf{1}]$. Since $M_{\lambda}$ is a composition factor and $M_{s_{\beta *} \cdot \lambda}$ is not a composition factor of $\operatorname{Ker} \varphi_{\beta}, \cap_{\beta \in S} \operatorname{Ker} \varphi_{\beta}$ has zero second socle level, i.e., $\bigcap_{\beta \in S} \operatorname{Ker} \varphi_{\beta}=M_{\lambda}=\operatorname{Soc}^{1}\left(H^{0}(\lambda)\right)$. By [6], for instance, we 
conclude that

$$
\operatorname{Soc}^{j}\left(H^{0}(\lambda)\right)=\bigcap_{\beta \in S} \varphi_{\beta}^{-1} \operatorname{Soc}^{j-1}\left(H^{0}\left(s_{\beta *} \cdot \lambda\right)\right), \quad \text { for } j \geq 1 .
$$

By that equality for $j=2$, we only need to show that $M_{s_{\alpha *} \cdot \lambda}$ is a composition factor of $\bigcap_{\beta \in S} \varphi_{\beta}^{-1}\left(M_{s_{\beta *} \cdot \lambda}\right)$, for each $\alpha \in S$, in order to complete the proof. Since $M_{s_{\alpha *} \cdot \lambda}$ is a composition factor of $H^{0}(\lambda)$ of multiplicity one, $M_{s_{\alpha *}}$ is a composition factor of the intersection if and only if it is a composition factor of each term $\varphi_{\beta}^{-1}\left(M_{s_{\beta *} \cdot \lambda}\right)$. If $\beta=\alpha$, that is so. If $\beta \neq \alpha$, then $M_{s_{\alpha *} \lambda}$ is not a composition factor of $H^{0}\left(s_{\beta *} \cdot \lambda\right)$, since $s_{\alpha *} \cdot \lambda \nless s_{\beta *} \cdot \lambda$; hence, $M_{s_{\alpha *} \cdot \lambda}$ is a composition factor of $\operatorname{Ker} \varphi_{\beta}$.

\section{The lower wall restriction on extensions.}

3.1. Let $A_{\chi}$ be the alcove which contains a $p$-regular weight $\chi$. Let $\gamma$ be a positive root. The following results are based on [6].

Definition. Suppose that the affine hyperplane $H_{\gamma, n p}$ contains a face $f$ of $A_{\chi} . f$ is a lower face if $\left(\gamma^{\nu}, \chi+\rho\right)>n p$.

Let $[f]=W_{p} \cdot f$ be the orbit of $f$ under $W_{p}$. Let $\mathscr{F}$ be the set of $W_{p}$-orbits of faces. $\mathscr{F}$ has $\operatorname{Card}(S)+1$ elements. Following the terminology of $[8]$, let

$$
\mathscr{L}(\chi)=\left\{[f] \in \mathscr{F} \mid[f] \cap A_{\chi} \text { is a lower face of } A_{\chi}\right\} .
$$

Call two alcoves adjacent if they share a face.

Suppose that $p$-regular weights $\chi$ and $\lambda$ are not in adjacent alcoves.

Proposition. If $\chi \in \operatorname{Ext}(\lambda)$ and $\chi<\lambda$, then $\mathscr{L}(\lambda) \subset \mathscr{L}(\chi)$.

Proof. Suppose that $H_{\gamma, n p}$ contains a lower face $f$ of $A_{\lambda}$. Take the non-zero intertwine $\varphi$ from $H^{0}(\lambda)$ to $H^{0}\left(s_{\gamma *} \cdot \lambda\right)$ [1]. The proposition of $[6, \S 2]$ and the annihilation property of the translation functor (cited in [6, §2]) imply that if $M_{\chi}$ is a composition factor of $\operatorname{Ker} \varphi$, then $[f] \in \mathscr{L}(\chi)$. It only remains to show that $M_{\chi}$ is a composition factor of $\operatorname{Ker} \varphi$. Since $A_{\lambda}$ and $A_{\chi}$ are not adjacent, $M_{\chi} \neq M_{s_{\gamma^{*}} \cdot \lambda}$. Since $M_{\chi}$ is a composition factor of $\operatorname{Soc}^{2}\left(H^{0}(\lambda)\right)$ and since $\varphi\left(\operatorname{Soc}^{2}\left(H^{0}(\lambda)\right)\right)$ is contained in $\operatorname{Soc}^{1}\left(H^{0}\left(s_{\gamma *} \cdot \lambda\right)\right)=M_{s_{\gamma^{*} \cdot} \cdot \lambda}, M_{\chi}$ must be a composition factor of $\operatorname{Ker} \varphi$.

The proposition, together with the Theorem of 2.2, gives the following estimate for $\operatorname{Ext}(\lambda)^{0}$. 
$\operatorname{Ext}(\lambda)^{0}$ is contained in the union of the $I_{W_{v}}$-orbits of the sets

(i)

(ii)

$$
\left\{s_{\beta *} \cdot \lambda \mid \beta \in S\right\},
$$

$$
\begin{gathered}
\left\{\chi \in \mathrm{SL} \cdot \lambda \cup \mathrm{SL}^{-1} \cdot \lambda \mid A_{\chi} \text { and } A_{\lambda} \text { are adjacent }\right\}, \\
\left\{\chi \in F^{v} \cap \mathrm{SL}^{-1} \cdot \lambda \mid \mathscr{L}(\chi) \subset \mathscr{L}(\lambda)\right\}, \\
\left\{\chi \in F_{v_{-1}} \cap \mathrm{SL} \cdot \lambda \mid \mathscr{L}(\lambda) \subset \mathscr{L}(\chi)\right\} .
\end{gathered}
$$

3.2. A conjectural bound for $\operatorname{Ext}(\lambda)^{0}$. Let $d(\lambda, \chi)$ count the number of hyperplanes dividing $\chi$ from $\lambda$, where a hyperplane is counted +1 if $\chi$ is on the positive side (i.e., $\chi$ is on the positive side of $H_{\gamma, n p}, \gamma \in R^{+}$, if $\left.\left(\gamma^{\nu}, \chi+\rho\right)>n p\right)$, and is counted -1 if $\chi$ is on the negative side [8]. With the work in [3] on Lusztig's conjective in mind, we add a conjectural restriction for $\chi$ to be an element of $\operatorname{Ext}(\lambda)^{0}$, namely, when $\chi \in \operatorname{Ext}(\lambda)^{0}$, $d(\lambda, \chi)$ is odd.

Conjectural bound on $\operatorname{Ext}(\lambda)^{0}$.

$\operatorname{Ext}(\lambda)^{0}$ is contained in the union of the orbits of the sets (i)-(iv) in 3.1 , where (iii) and (iv) are restricted further by the requirement that $d(\lambda, \chi)$ be odd.

For groups of rank 2, $\operatorname{Ext}(\lambda)^{0}$ equals the conjectured bound $(\lambda$ generic). For groups of rank $>2$, we have no information about whether $\operatorname{Ext}(\lambda)^{0}$ equals the conjectured bound.

\section{Further conjectures for $m \operatorname{Ext}(\lambda)^{0}$.}

4.1. Let $\operatorname{Soc}_{2}=\operatorname{Soc}^{2} / \operatorname{Soc}^{1}$. Let $\lambda$ be a $p$-regular weight in $V^{v}$ and let $y_{0}$ be the longest word in $W_{v}$. Assume that $\lambda$ and $y_{0} \cdot \lambda$ are generic weights.

DEFINITIONS.

$$
\begin{aligned}
& m \operatorname{Ext}^{+}(\lambda)^{0}=\left\{(\chi, n) \in m \operatorname{Ext}(\lambda)^{0} \mid \chi \in \mathrm{SL}^{-1} \cdot \lambda\right\}, \\
& m \operatorname{Ext}^{-}(\lambda)^{0}=\left\{(\chi, n) \in m \operatorname{Ext}(\lambda)^{0} \mid \chi \in \mathrm{SL} \cdot \lambda\right\} .
\end{aligned}
$$

$m \operatorname{Ext}^{-}(\lambda)^{0}$ coincides with $X\left[\operatorname{Soc}_{2}\left(H^{0}(\lambda)\right)\right]$.

Duality conjecture. $y_{0} \cdot m \operatorname{Ext}(\lambda)^{0}=m \operatorname{Ext}\left(y_{0} \cdot \lambda\right)^{0}$.

Proposition. If the duality conjecture is valid, then

$$
m \operatorname{Ext}(\lambda)^{0}=X\left[\operatorname{Soc}_{2}\left(H^{0}(\lambda)\right)\right] \cup y_{0} \cdot X\left[\operatorname{Soc}_{2}\left(H^{0}\left(y_{0} \cdot \lambda\right)\right)\right] .
$$


Proof. We have

$$
\begin{aligned}
m \operatorname{Ext}(\lambda)^{0} & =m \operatorname{Ext}^{-}(\lambda)^{0} \cup m \operatorname{Ext}^{+}(\lambda)^{0} \\
& =m \operatorname{Ext}^{-}(\lambda)^{0} \cup y_{0} \cdot m \operatorname{Ext}^{-}\left(y_{0} \cdot \lambda\right)^{0} \quad \text { (using the hypothesis) } \\
& =X\left[\operatorname{Soc}_{2}\left(H^{0}(\lambda)\right)\right] \cup y_{0} \cdot X\left[\operatorname{Soc}_{2}\left(H^{0}\left(y_{0} \cdot \lambda\right)\right)\right] .
\end{aligned}
$$

We note that $y_{0}$ carries the bound for $\operatorname{Ext}(\lambda)^{0}$, given in 3.1 , into the bound for $\operatorname{Ext}\left(y_{0} \cdot \lambda\right)^{0}$. That follows from the formulas (i)-(iii) below. Let $v=\bigcap_{\alpha \in S} H_{\alpha, n_{\alpha} p}$ and $v_{+1}=\bigcap_{\alpha \in S} H_{\alpha,\left(n_{\alpha}+1\right) p}$. Let $y_{+}$be the element of $W_{v_{+1}}$ corresponding to the element $y$ of $W_{v}$.

(i) $y_{0} \cdot I_{W_{v}} \cdot \chi=I_{W_{v_{+}}} \cdot y_{0} \cdot \chi$; more precisely, $y_{0} \cdot I_{y} \cdot \chi=I_{\left(y_{0} y y_{0}\right)_{+}} \cdot$ $y_{0} \cdot \chi$

(ii) $y_{0} \cdot\left(F_{v_{-1}} \cap \mathrm{SL} \cdot \lambda\right)=F^{v_{+}} \cap \mathrm{SL}^{-1} \cdot \lambda ; \quad y_{0} \cdot\left(F^{v} \cap \mathrm{SL}^{-1} \cdot \lambda\right)=$ $F_{v} \cap \mathrm{SL} \cdot \lambda$.

(iii) $y_{0} \cdot s_{\beta^{*}} \cdot \lambda=I_{\left(y_{-w_{0}(\beta)}\right)_{+}} \cdot s_{-w_{0}(\beta) *} \cdot y_{0} \cdot \lambda$.

(i) may be verified at $y=y_{\beta}$. For (iii), we have $y_{0} s_{\beta *} \cdot \lambda=s_{w_{0}(\beta) *} \cdot y_{0}$ $\cdot \lambda=s_{-w_{0}(\beta)}^{*} \cdot y_{0} \cdot \lambda_{1}$ and $I_{\left(y_{-w_{0}(\beta)}\right)+s_{-w_{0}(\beta)}} \cdot \chi=s_{-w_{0}(\beta)}^{*} \cdot \chi$, for $\chi \in V^{v_{+1}}$. Since $y_{0} \cdot \lambda \in V^{v_{+}}$when $\lambda \in V^{v}$, the formula is verified.

4.2. We give a conjectural formula for $m \operatorname{Ext}(\lambda)^{0}$ in terms of the second and $N$ th socle levels of $H^{0}(\lambda)$.

We will say that the radical and socle series for $H^{0}(\lambda)$ coincide if $\operatorname{Soc}^{j}\left(H^{0}(\lambda)\right)=\operatorname{Rad}^{N+1-j}\left(H^{0}(\lambda)\right)$ for $j=1, \ldots, N+1$. If $M$ is a module, let $M^{\text {tr }}$ be the transposed dual module (see [6], for instance). If $M_{1}$ is a submodule of $M$, let $M_{1}^{\perp}=\left\{f \in M^{\text {tr }} \mid f\left(M_{1}\right)=0\right\}$.

Proposition. If the duality conjecture for $m \operatorname{Ext}(\lambda)^{0}$ is valid and if the radical and socle series for $H^{0}(\lambda)$ coincide, then

$$
m \operatorname{Ext}(\lambda)^{0}=X\left[\operatorname{Soc}_{2}\left(H^{0}(\lambda)\right)\right] \cup J_{w_{0}}^{-1} \cdot X\left[\operatorname{Soc}_{N}\left(H^{0}(\lambda)\right)\right] .
$$

Proof. In general,

$$
H^{0}(\lambda)^{\operatorname{tr}} \cong H^{N}\left(w_{0} \cdot \lambda\right)
$$

and

$$
\left[\operatorname{Rad}^{j}\left(H^{0}(\lambda)\right)\right]^{\perp} \cong \operatorname{Soc}^{j}\left(H^{N}\left(w_{0} \cdot \lambda\right)\right) .
$$

By the second hypothesis,

$$
\operatorname{Rad}^{j}\left(H^{0}(\lambda)\right)=\operatorname{Soc}^{N+1-j}\left(H^{0}(\lambda)\right) .
$$

Hence,

$$
\operatorname{Soc}^{1}\left(H^{N}\left(w_{0} \cdot \lambda\right)\right) \cong \operatorname{Soc}^{N}\left(H^{0}(\lambda)\right)^{\perp},
$$


and

$$
\operatorname{Soc}^{2}\left(H^{N}\left(w_{0} \cdot \lambda\right)\right) \cong \operatorname{Soc}^{N-1}\left(H^{0}(\lambda)\right)^{\perp} .
$$

Taking quotients, we have

$$
\begin{aligned}
\operatorname{Soc}_{2}\left(H^{N}\left(w_{0} \cdot \lambda\right)\right) & \cong \operatorname{Soc}^{N-1}\left(H^{0}(\lambda)\right)^{\perp} / \operatorname{Soc}^{N}\left(H^{0}(\lambda)\right)^{\perp} \\
\cong & {\left[\operatorname{Soc}^{N}\left(H^{0}(\lambda)\right) / \operatorname{Soc}^{N-1}\left(H^{0}(\lambda)\right)\right]^{\text {tr }}=\operatorname{Soc}_{N}\left(H^{0}(\lambda)\right)^{\text {tr }} . }
\end{aligned}
$$

Hence, by the proposition in 4.1, it will suffice to show that

$$
y_{0} \cdot X\left[\operatorname{Soc}_{2}\left(H^{0}\left(y_{0} \cdot \lambda\right)\right)\right]=J_{w_{0}}^{-1} \cdot X\left[\operatorname{Soc}_{2}\left(H^{N}\left(w_{0} \cdot \lambda\right)\right)\right],
$$

since $\operatorname{tr}$ leaves semisimple modules unaltered. Since $J_{w_{0}} \cdot y_{0}=I_{y_{0}}$, the last equality is just the property of alcove transition.

REMARK. Let $y_{0}^{\prime}$ be the longest word in $W_{v^{\prime}}$. On $V^{v^{\prime}}, J_{w_{0}}^{-1}$ may be computed by: $J_{w_{0}}^{-1}=y_{0}^{\prime}$.

\section{REFERENCES}

[1] $\mathrm{H}$. Andersen, On the structure of the cohomology of line bundles on $G / B$, J. Algebra, 71 (1981), 245-258.

[2] _ On the generic structure of cohomology modules for semi-simple algebraic groups, Trans. Amer. Math. Soc., 295 (1986), 397-415.

[3] _ Jantzen's Filtrations of Weyl Modules, Math. Z., 194 (1987), 127-142.

[4] Cline, Parshall, Scott, and Van der Kallen, Rational and generic cohomology, Inventiones Math., 39 (1977), 143-163.

[5] Cline, Parshall, and Scott, On injective modules for infinitesimal algebraic groups, I, J. London Math. Soc., (2) 31 (1985), 277-291.

[6] S. Doty and J. Sullivan, The submodule structure of Weyl modules for $\mathrm{SL}_{3}$, J. Algebra, 96 (1985), 78-93.

[7] On the structure of the higher cohomology modules of line bundles on $G / B$, to appear, J. Algebra.

[8] G. Lusztig, Hecke algebras and Jantzen's generic decomposition patterns, Advances in Math., 37 (1980), 121-164.

[9] J.-c. Ye, Filtrations of principal indecomposable modules of Frobenius kernels of reductive groups, Math. Z., 189 (1985), 515-527.

[10] H. Andersen, Extensions of modules for algebraic groups, Amer. J. Math., 106 (1984), 489-504.

Received June 23, 1986 and in revised form December 12, 1986.

UNIVERSITY OF WASHINGTON

SEATTLE, WA 98195 
